\title{
Vulvar Pruritus: Variability of Clinical Evaluation and Management
}

\author{
Sarah L Bedell, Ashli A Lawson, William F Griffith and Claudia L Werner* \\ Department of Obstetrics and Gynecology, University of Texas Southwestern Medical Center, USA
}

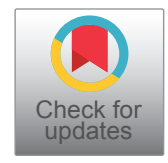

*Corresponding author: Claudia L Werner, MD, Department of Obstetrics and Gynecology, University of Texas Southwestern Medical Center, 5323 Harry Hines Boulevard, Dallas, TX, 75390-9032, USA, Tel: 214-648-3662, Fax: 214$648-9028$

\begin{abstract}
Objective: We characterize the evaluation and initial management of patients with vulvar pruritus, including elements of history-taking, physical examination, laboratory testing, and treatments. We propose an algorithm for approaching this common clinical problem in a systematic way.

Methods: A retrospective chart review of patients with vulvar pruritus who presented to Gynecology or Vulvology Clinic at Parkland Health and Hospital System in 2012 informed this descriptive study.

Results: A total of 46 patients aged 19 to 70 years presented with a primary symptom of vulvar itching, of which $41 \%$ had additional irritative vulvar complaints. The average duration of symptoms was 24 months; $67 \%$ had used at least one self-treatment unsuccessfully prior to their visit. Only $13 \%$ of patients received a non-genital skin examination; $46 \%$ received a vaginal culture, and $20 \%$ underwent vulvar biopsy. The most common diagnoses were "nonspecific" $(39 \%)$, and "dermatitis" (22\%). The most common treatment prescribed was a topical steroid $(78 \%)$. Vulvar skin care counseling was documented for $67 \%$ of patients. At 18 months, $46 \%$ were lost to follow up and of those who returned, $48 \%$ reported resolution of initial vulvar symptoms.
\end{abstract}

Conclusions: The assessment and treatment of vulvar pruritus is variable. An algorithm for the evaluation of this common clinical complaint is proposed.

\section{Keywords}

Vulvar itching, Vulvar irritation, Vulvar pruritis

\section{List of Abbreviations and Acronyms}

DNA: Deoxyribonucleic Acid; ICD-9: International Classification of Diseases, $9^{\text {th }}$ Revision; HRT: Hormone Replacement Therapy; PHHS: Parkland Health and Hospital System; VI: Vulvar Irritation; VP: Vulvar Pruritus, CG/CT: gonorrhea/Chlamydia

\section{Introduction}

Vulvar pruritus (VP), along with other symptoms of vulvar irritation (VI), is a common complaint for which women present to obstetrician-gynecologists and other primary care providers. In addition to itching, $\mathrm{VI}$ includes a sensation of irritation, chafing, pain or burning, for which women commonly self-treat with increased washing and over-the-counter hygiene or medicinal products. With a $\$ 3$ billion feminine care product industry, women have become regular users of products that contain known vulvar irritants [1-5]. Paradoxically, products meant to treat VP/VI can cause vulvar dermatitis and worsen preexisting symptoms [6]. While the exact prevalence of vulvar dermatitis is unknown, it has been shown to occur in up to 20 to $64 \%$ of patients with chronic vulvar symptoms $[7,8]$. It appears that both contact and irritant dermatitis play more significant roles than infection in the etiology of vulvar complaints $[4,5,9,10]$.

Despite being common, vulvar symptoms can be challenging both diagnostically and therapeutically due to a lack of a standardized clinical approach. Additional obstacles in the management of VP/VI include the fact that no one specialty claims or prioritizes vulvar disease, and evaluation of these complaints is not always included in training programs $[5,11]$. While a small amount of expert opinion and recommendations are available, there are no widely practiced or standardized guidelines for clinicians. Additionally, there is little data regarding the relevance of personal hygiene habits and their impact on vulvar disease, and a targeted history to elicit these behaviors is not consistently obtained [12].

Citation: Bedell SL, Lawson AA, Griffith WF, Werner CL (2018) Vulvar Pruritus: Variability of Clinical Evaluation and Management. Int J Womens Health Wellness 4:084. doi.org/10.23937/24741353/1510084

Accepted: November 28, 2018: Published: November 30, 2018

Copyright: (c) 2018 Bedell SL, et al. This is an open-access article distributed under the terms of the Creative Commons Attribution License, which permits unrestricted use, distribution, and reproduction in any medium, provided the original author and source are credited. 
Moreover, vulvar dermatoses can be difficult to recognize clinically as findings on exam may be subtle $[5,13]$. We suspected that both the assessment and management of patients with vulvar pruritus and related symptoms are inconsistent at our own institution, and this may be the case elsewhere. The lack of a systematic approach likely contributes to delayed diagnosis, multiple clinical visits, and patient or physician frustration. We characterize patients presenting with VP and other VI symptoms in a specialized clinic setting, describe provider approaches to patients with these complaints, and propose an algorithm (Appendix) to guide the evaluation and initial management based on these findings $[14,15]$.

\section{Methods}

With Institutional Review Board approval from the University of Texas Southwestern Medical Center, we performed a retrospective chart review of all female patients 18 to 80 years of age who presented to Parkland Health and Hospital System (PHHS) from January to December, 2012 and whose charts were coded with ICD-9 698.1: pruritus of genital organs. Of these patients, we selected those who presented for an initial visit to the general Gynecology Clinic or the specialized Vulvology Clinic within the Gynecology Clinic. Charts were reviewed to confirm that the diagnostic code pertained to an actual vulvar complaint. We tabulated demographic and historic information including age, symptom duration, presence of additional irritative symptoms (irritation, burning, or pain), whether associated vaginal complaints (including discharge) were present, as well as the use of any self-treatments prior to the initial visit. We recorded whether the provider questioned the patient specifically regarding vulvar hygiene habits. Elements of physical examination were recorded including whether the patient received targeted vulvar, vaginal, non-genital skin, and oral mucosa exams. Any testing obtained was recorded including saline wet prep of vaginal secretions, vaginal fungal or aerobic culture, gonorrhea and Chlamydia DNA polymerase testing, and vulvar skin biopsy. We tabulated up to three consecutive treatments each patient received and whether the patient was counseled regarding vulvar skin care recommendations as part of management. The presumptive clinical diagnosis was recorded as well as whether the diagnosis changed by 18 months of surveillance. Charts were also reviewed to determine the number of additional visits the patient had for the same complaint by 18 months of follow up, and whether their symptoms were persistent, improved or resolved at their latest visit. Chart abstraction was performed by two of the authors (SLB and AAL) with reconciliation of any discrepancies by repeat, joint chart review. Analysis of the selected parameters was performed using simple descriptive statistics.

\section{Results}

During 2012, a total of 1,175 female patient visits at PHHS were coded as ICD-9 698.1: pruritus of genital organs; this included all outpatient and Emergency Department encounters. Of these patients, only 123 patients were seen in the Gynecology or Vulvology Clinics. Patients whose vulvar complaint was not the primary purpose of the visit, who had a specifically vaginal complaint, or who were not seen for an initial evaluation (i.e. surveillance visits only) were excluded $(n=77)$. Information was tabulated for the remaining 46 patients seen for initial evaluation of a vulvar complaint. The average age of these patients was 46 years with a range of 19 to 70 years. Patients were seen in the general Gynecology Clinic (22\%) or a specialized Vulvology Clinic $(78 \%)$ at PHHS. Symptom duration prior to presentation ranged from 1 to 120 months, with an average of 24 months. Concurrent additional complaints of vulvar burning, pain, irritation, or vaginal discharge were reported in $41 \%$ of patients while $30 \%$ also reported vaginal itching. Inquiry regarding vulvar hygiene habits such as methods of genital cleansing, soaps and/or laundry detergents used (names, amounts, frequency of use) was documented for $70 \%$ at initial patient encounters.

For $91 \%$ of patients, there was documentation of prior self-treatment; of these, $67 \%$ of patients used one, $25 \%$ used two, and one patient (4\%) used three products before initial presentation. The most commonly used self-treatments were topical antifungals (36\%), topical steroids (29\%) or topical estrogens (14\%) acquired previously (Table 1). Regarding physical examination, all patients received a vulvar examination by unaided or magnified inspection, $96 \%$ underwent a vaginal speculum examination and $13 \%$ underwent a documented non-genital skin examination. During initial evaluation, testing included a saline wet prep of vaginal secretions for $65 \%$ of patients, an aerobic vaginal culture for $46 \%$, gonorrhea/Chlamydia testing for $46 \%$ and a vulvar biopsy for $20 \%$ of patients.

The most common initial clinical diagnoses were non-specific, including a restatement of patient symptoms (39\%), irritant or contact dermatitis (22\%), or specific vulvar dermatoses including lichen sclerosus, lichen planus or lichen simplex chronicus (17\%) (Table

Table 1: Treatment(s) used by patients with complaints of vulvar itching prior to their initial visit.

\begin{tabular}{|l|l|}
\hline Topical anti-fungal & $10(36 \%)$ \\
\hline Topical steroid & $8(29 \%)$ \\
\hline Topical estrogen & $4(14 \%)$ \\
\hline Oral anti-fungal & $3(11 \%)$ \\
\hline Lidocaine gel & $2(7 \%)$ \\
\hline Feminine anti-itch cream & $2(7 \%)$ \\
\hline Benadryl cream & $1(4 \%)$ \\
\hline Metronidazole & $1(4 \%)$ \\
\hline Topical emollient & $1(4 \%)$ \\
\hline
\end{tabular}

All values are listed as $n(\%)$. 
Table 2: Clinical diagnosis at the patient's initial visit.

\begin{tabular}{|l|l|}
\hline Non-specific/Restatement of symptoms & $18(39 \%)$ \\
\hline Irritant or contact dermatitis & $10(22 \%)$ \\
\hline Dermatosis & $8(17 \%)$ \\
\hline Infectious other & $3(7 \%)$ \\
\hline Dermatitis or candida & $3(7 \%)$ \\
\hline Neoplasia & $2(4 \%)$ \\
\hline Candida & $1(2 \%)$ \\
\hline Normal exam & $1(2 \%)$ \\
\hline
\end{tabular}

All values are listed as n (\%). "Infectious other" refers to a diagnosis of infections not including candida (bacterial vaginosis, folliculitis, HSV, etc). "Neoplasia" represents both vulvar dysplasia and benign vulvar growths or lesions.

Table 3: Treatment(s) prescribed by providers at the patient's initial visit.

\begin{tabular}{|l|l|}
\hline Topical steroid & $31(78 \%)$ \\
\hline Topical anti-fungal & $8(20 \%)$ \\
\hline No treatment & $6(13 \%)$ \\
\hline Lidocaine gel & $4(10 \%)$ \\
\hline Oral anti-fungal & $3(8 \%)$ \\
\hline Hydroxyzine & $3(8 \%)$ \\
\hline Topical estrogen & $1(3 \%)$ \\
\hline Metronidazole & $1(3 \%)$ \\
\hline Vaginal moisturizer & $1(3 \%)$ \\
\hline
\end{tabular}

All values are listed as $\mathrm{n}(\%)$. Patients can be included in more than one category.

2). Of note, for $7 \%$ of patients, providers were unable to distinguish if a patient's symptoms were the result of a dermatitis or Candida. Patients received information regarding recommended vulvar care measures in the form of counseling and/or a printed handout at $67 \%$ of initial encounters. Approximately $87 \%$ were prescribed at least one treatment during their initial evaluation; of these, $33 \%$ received two treatments and $2 \%$ received three. The most commonly prescribed treatments were topical steroids (78\%), topical anti-fungals $(20 \%)$ and topical lidocaine (10\%) (Table 3).

Within 18 months, 54\% of patients had encounters for vulvar symptom reassessment. Of these patients, $11 \%$ received a change in diagnosis. Of the 9 patients who underwent vulvar biopsy, 8 had a histologic diagnosis consistent with their clinical diagnosis; four showed lichen sclerosus, four demonstrated findings consistent with contact or irritant dermatitis, and one was consistent with a hidradenoma papilliferum, a benign apocrine tumor. Patients had an average of two return visits (range 0 to 8 ) for complaints of vulvar itching in the following 18 months. At 18 months, $20 \%$ of patients experienced recurrent or persistent symptoms, $32 \%$ had improvement, and $48 \%$ of patients reported resolution of their symptoms.

\section{Discussion}

Complaints of vulvar itching and/or irritation, particularly itching, are common and affect a wide age range; the duration of symptoms at presentation is highly variable. They often lead to multiple outpatient visits for evaluation and treatment. Over 1,100 visits in one year to our hospital system were related to such vulvar complaints. A detailed, focused history and knowledge of the common causes of vulvar irritation inform the proper diagnosis and management of these patients. In our experience, patients often assume that their symptoms are due to inadequate hygiene or infection, triggering escalating hygiene practices that in turn exacerbate irritation and lead to ineffective self-treatment. This study is consistent with previously published investigations demonstrating that patients frequently misdiagnose themselves, leading to the unindicated or ineffective use of nonprescription antifungals, anti-itch creams, and topical antibiotics [16]. For example, more than a third of our patients self-treated with anti-fungals prior to presentation. Without a careful history, these potentially contributory behaviors can be overlooked and go unaddressed. Symptom duration is often prolonged, with an average time of 2 years reported by our patients. Our study confirms that the majority of vulvar itching and related complaints are due to noninfectious etiologies, and treatment with topical steroids are most often prescribed [10] followed by antifungals. Aerobic cultures to identify fungal vulvovaginitis not detected by vaginal wet prep did not change the diagnosis in any case at initial encounter. Likewise, vulvar biopsies failed to alter the initial diagnosis. This implies that these two tests can be reserved for subsequent evaluation of refractory cases.

The strengths of our study include the careful and adjudicated data collection completed by two of the authors. The restriction of patients to those seen under one specific diagnostic code commonly used at our institution allowed focus on patients presenting specifically for the vulvar symptoms addressed by this study. Limitations include a relatively small number of study subjects. The inclusion of patients within only one diagnostic code for genital pruritus likely excluded a much larger number of patients coded for other specific vulvar complaints such as vulvar irritation, pain, and burning, or as having vaginal, vulvovaginal, or other coexistent gynecologic complaints. Indeed, approximately two-thirds of patients coded for vulvar pruritus were eliminated from the study due to miscoding of the primary complaint in the electronic medical record. Limiting this review to visits within the Department of Gynecology (including a specialty clinic for vulvar complaints) surely underestimates the number of visits to all types of providers for vulvar pruritus and irritation as well as the variation in evaluation and management that patients experience within this hospital system. Indeed, approximately ten times more visits coded as vulvar pruritis took place outside the Gynecology or Vulvology Clinics at PHHS. Treatments beyond the maximum of three per patient were not recorded during the study time period. Furthermore, a co-existing diagnosis of diabetes was not elicited. As 


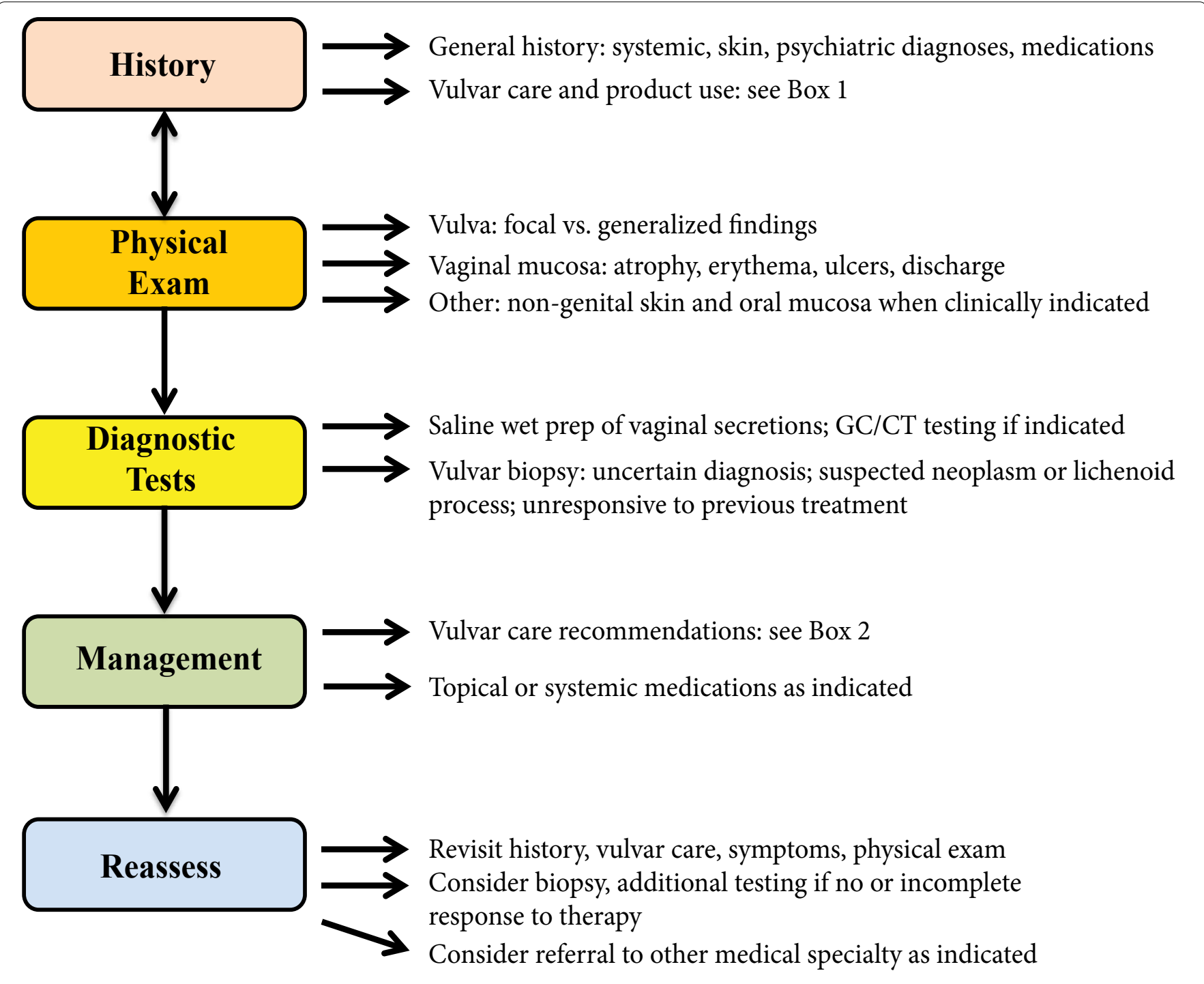

Figure 1: Proposed algorithm for the evaluation and initial management of patients with irritative vulvar symptoms (Box 1 and Box 2).

such, the degree to which this affects patient symptoms and number of clinic visits was not studied, however this likely does contribute to persistent symptoms of vulvar pruritis, especially via infectious agents.

\section{Conclusions}

The clinical assessment and monitoring of patients with vulvar pruritus and additional irritative vulvar complaints is variable, even in a specialized clinical setting. The standardization of clinical evaluation and initial management of this common complaint is warranted in order to study best approaches to initial evaluation and management. A clinical algorithm is proposed in the Appendix that follows as a potential move toward this goal, encouraging a thoughtful, detailed, organized approach that will more efficiently and accurately aid the diagnosis, management, and study of vulvar pruritus and other irritative complaints (Figure 1).

\section{Declaration of Interests}

The authors have no conflicts of interest to declare. The authors have no financial support disclosures.

\section{References}

1. Schlosser BJ (2010) Contact dermatitis of the vulva. Dermatol Clin 28: 697-706.

2. Trager JD (2005) What's your diagnosis? Acute vulvar erythema, edema, and pruritus in a young woman. J Pediatr Adolesc Gynecol 18: 275-280.

3. Foote CA, Brady SP, Brady KL, Clark NS, Mercurio MG (2014) Vulvar dermatitis from allergy to moist flushable wipes. J Low Genit Tract Dis 18: E16-E18.

4. Hoang MP, Reutter J, Papalas JA, Edwards L, Selim MA (2014) Vulvar inflammatory dermatoses: An update and review. Am J Dermatopathol 36: 689-704.

5. Margesson LJ (2006) Vulvar disease pearls. Dermatol Clin 24: $145-155$.

6. Erekson EA, Martin DK, Brousseau EC, Yip SO, Fried TR (2014) Over-the-counter treatments and perineal hygiene in postmenopausal women. Menopause 21: 281-285.

7. Stewart EJC, Wojnarowska F, Marren PM (1995) Experience in a clinic dedicated to the study of vulvar disorders. Br J Obs Dermatol 133: 31-32.

8. Fischer G, Spurret B, Fischer A (1995) The chronically symptomatic vulva: Aetiology and management. $\mathrm{Br} J$ Obstet Gynaecol 102: 773-779. 
9. Crone AM, Stewart EJ, Wojnarowska F, Powell SM (2000) Aetiological factors in vulvar dermatitis. J Eur Acad Dermatol Venereol 14: 181-186.

10. Nyirjesy P, Peyton C, Weitz MV, Mathew L, Culhane JF (2006) Causes of chronic vaginitis: Analysis of a prospective database of affected women. Obstet Gynecol 108: 1185-1191.

11. Edwards L (2010) Dermatologic causes of vaginitis: A clinical review. Dermatol Clin 28: 727-735.

12. Marin M-G, King R, Sfameni S, Dennerstein GJ (2000) Adverse behavioral and sexual factors in chronic vulvar disease. Am J Obstet Gynecol 183: 34-38.
13. Williams JW, Hoffman BL (2012) Williams gynecology. (2 $2^{\text {nd }}$ edn), McGraw-Hill Education, New York.

14. Burrows LJ, Shaw HA, Goldstein AT (2008) The vulvar dermatoses. J Sex Med 5: 276-283.

15. Farage MA (2005) Vulvar susceptibility to contact irritants and allergens: A review. Arch Gynecol Obstet 272: 167172.

16. Nyirjesy P, Weitz M, Grody M, Lorber B (1997) Overthe-counter and alternative medicines in the treatment of chronic vaginal symptoms. Obstet Gynecol 90: 50-53.

Box 1: Points to review with patients when obtaining a history related to irritative vulvar symptoms.

Medical Conditions: Diabetes, immune suppression, liver or renal disease, chronic diarrhea, Crohn's disease, psychiatric diagnoses

Hormonal Status: Pregnant, breastfeeding, menopausal, hormone therapy

Non-genital Skin Conditions: Dry skin, eczema, psoriasis, rosacea, tinea, candidiasis, hidradenitis, lichen planus, lichen sclerosus

\section{Bathing/Hygiene Habits:}

Frequency, water temperature, bath vs. shower, soaps (liquid vs. bar, fragrances, other chemical additives); use of bath, dishwashing, or laundry products on body or in bath water (dishwashing liquid, bubble bath, Epsom salts, bleach); mechanical scrubbing (wash cloths, mesh poufs, loofas); shaving or other methods of genital hair removal; use of wet wipes

\section{Product Use in Genital Area:}

Menstrual and continence pads, feminine hygiene products (douches, sprays, washes), feminine anti-itch products, baby or adult cleansing wipes, antibiotic or other creams or ointments, perfumes, mouthwash, hydrogen peroxide, rubbing alcohol, others

\section{Continence Problems:}

Chronic urine leakage, fecal incontinence, fistulae

\section{Clothing (underwear and outerwear):}

Tight-fitting pants or underwear; occlusive, nonabsorbent (usually synthetic) fabrics such as Lycra or Spandex, exercise outfits, bathing suits

\section{Laundry Practices:}

Front versus top-loading machines (front-loaders require less detergent), amount of detergent used per load, detergent brand, fragranced vs. fragrance/dye free, fabric softeners, bleach, dryer sheets, others

Box 2: Vulvar care recommendations for patients.

\section{General health:}

- Optimize medical or skin conditions that may be contributing to vulvar irritation.

- $\quad$ Address obesity as a potential factor in vulvar skin health (more moisture, mechanical abrasion, tight clothing).

Breaking the itch-scratch cycle:

- Cut finger nails short temporarily.

- Wear white cotton gloves (washed) to bed - can be garden gloves.

- $\quad$ Take diphenhydramine $25 \mathrm{mg}$ (Benadryl) at bedtime if safe to do so.

- A thin coating of white, plain petrolatum jelly can be applied to the vulva if skin is very dry or irritated.

\section{Genital bathing habits:}

- Stop frequent or excessive washing/rinsing.

- Avoid prolonged sitting in tub water.

- Use warm water in shower or tub, never hot.

- Wash/rinse genitals last to remove other products used on hair \& body.

- Use unscented or hypoallergenic bar bath soaps only, well-diluted with water, no more than once a day, less if possible; discontinue liquid, scented, anti-bacterial soaps. Soaps marketed for sensitive skin are usually a good choice.

- Discontinue douching. 
- Do not use washcloths, scrubbers, etc. to wash.

- Dry thoroughly before dressing; pat genitals dry gently (no rubbing), or use a blower dryer on cool setting.

- Discontinue genital shaving, waxing, or use of depilatories.

\section{STOP use of products known to cause genital irritation:}

- Wet wipes for babies and adults.

- $\quad$ Bath additives including bubble bath, scented bath oils, bath or Epsom salts, rubbing alcohol, bleach, or dishwashing liquids.

- Hydrogen peroxide.

- Topical antibiotics.

- Over-the-counter anti-itch or other creams.

- $\quad$ Feminine hygiene washes or sprays.

- Deodorant-containing menstrual pads or tampons.

- Colored or scented toilet paper.

Sexual intercourse and contraception:

- Try non-latex condoms if latex sensitivity is suspected.

- Discontinue scented or otherwise enhanced lubricants.

- $\quad$ Consider cooking oils or vegetable shortening as alternative lubricants (may weaken latex condoms).

\section{Continence:}

- Tell your provider about any urinary or fecal incontinence issues you are having.

\section{Clothing:}

- Wear plain cotton underwear, preferably white.

- Underwear and outer clothing should be made of natural fibers, not synthetics that trap moisture.

- $\quad$ All clothing below the waist (especially pants) should be worn as loosely as possible.

- Wear skirts or dresses when possible; wear a house dress or bathrobe at home.

- Take off underwear if possible at home and in bed.

\section{Laundry products:}

- Use laundry detergents without fragrances, bleach, dyes, and other additives. These often come in white boxes or bottles.

- Use $1 / 4$ to $1 / 2$ the amount of detergent recommended; pay attention to markings on the measuring cap. Detergents are highly concentrated, and using more than this does not get clothes cleaner. If a frontloading machine is used, use only a couple of tablespoons of detergent per load. If you see more than a thin layer of suds in the wash cycle, you are using too much detergent.

- Consider a double rinse for clothing worn below the waist.

- Do not use dryer sheets or fabric softeners for clothing that is worn below the waist.

\section{Appendix:}

\section{Algorithm for the Evaluation of Vulvar Pruritus/ Irritation (Figure 1)}

We propose an algorithm to standardize the evaluation and initial management of irritative vulvar symptoms in patients presenting in any clinical setting. A detailed history (Box 1) should ascertain whether complaints are vulvar, vaginal, or both. Patients commonly misrepresent their vulvar complaints as "vaginal". Patients rarely disclose all bathing behaviors (including frequency, use of scrubbing aids like wash clothes), soaps, or hygiene products being used without specific questioning. Laundry product use, particularly those with scents and other additives, and amount used per load is also important. Use of laundry products in excess of what is recommended is very common, leaving residual detergents and softeners in clothing that readily leach onto vulvar skin. Use of antibiotic creams, anti-itch products, and pre-moistened wipes should be investigated. The unexpected, sometimes astonishing products and remedies patients report using should be explored. Moreover, if a history of diabetes is elicited, adequacy of blood sugar control must be assessed. The progression from the "History" to the "Physical Exam" is denoted by a double arrow in the algorithm, signifying the complementary nature of these components.

A thorough, consistent physical examination is essential. With adequate lighting, the mons pubis, vulva, perineum, and perianal skin should be examined thoroughly for focal lesions, erythema, abnormal pigmentation, rashes, and excoriations. Attention to 
specific patterns of findings, including whether localized vs. generalized and symmetric vs. asymmetric, can help distinguish between various vulvar dermatoses while screening for potential neoplastic, autoimmune, and infectious lesions. Generalized erythema can indicate the use of various contact irritants and allergens, including soaps, cleansing wipes, lotions, detergents, or products that remove protective oils from the skin and cause irritation. Symmetrical vulvar erythema that involves only the dependent, central edges of the labia majora, sparing the inner and outer labia, often indicates a reaction to a laundry product, especially if there is also involvement of the medial thighs, inguinal folds, mons pubis, perianal or gluteal regions. Thickened, "leathery" skin with depigmentation or a dusky grey or silver appearance due to hyperkeratosis often signifies chronic scratching rather than an acute process. The irritant that initiated the itch-scratch cycle in the past may no longer be at play once this cycle becomes established. Further questioning in response to this finding may reveal a disruption of sleep due to the severity of itching, which often worsens at night. The presence of swelling with vesicles or erosions signifies a severe, acute allergic dermatitis, and secondary infection with findings of pustules, crusting or fissures should also be noted [5]. Lichen sclerosus can be distinguished with its typical patterns of distribution, scarring or narrowing of the introitus, distorted vulvar architecture, skin thinning, and the presence of white, atrophic plaques [13]. Findings consistent with the various forms of lichen planus should be noted such as brightly erythematous erosions with white striations involving the vulva, vestibule or vagina.

A vaginal speculum examination is recommended during the initial evaluation of vulvar complaints. The presence of lesions, vaginal discharge, and evidence of estrogen effect (rugae, pink, moist mucosa) vs. atrophy (loss of rugae, mucosal pallor, lack of moisture) should be noted. Findings may be dramatic and unexpected. For example, the presence of erythematous erosions with a copious, yellow discharge with or without a fragile vaginal mucosa may indicate lichen planus [14]. Finally, examination of non-genital skin and oral mucosa should be performed. The presence of oral ulcerations, erosions, or white striations (suggestive of lichen planus) may indicate the correct vulvar diagnosis [14].

Additional evaluation may include several simple tests. A saline wet prep of vaginal secretions and assessment of vaginal $\mathrm{pH}$ can be helpful in correctly diagnosing a contributory vaginitis. Testing for gonorrhea and/ or Chlamydia infection may be indicated. Aerobic vaginal culture (without Gram stain) was obtained in $46 \%$ of our patients to detect clinically significant levels of fungal organisms that may be missed on a wet prep. While this test, in our experience, never indicated candidiasis or subsequently altered treatment, this may be due to recent use of topical anti-fungals by the patient and should still be considered as part of the initial workup. Fungal culture and sensitivity can be used more selectively when resistant yeast infection is suspected due to treatment failures. When atopic dermatitis is suspected, referral for patch testing may be performed to reveal potential unsuspected allergens $[14,15]$.

Vulvar biopsy should be considered when neoplasia or a lichenoid process is suspected, or when a clinical diagnosis is not strongly suggested by the history and physical examination. This also should be considered for patients that do not respond to treatment. Biopsies at or near the clitoris should be performed sparingly and only by those experienced in the assessment of vulvar conditions.

Management of vulvar symptoms always includes extensive counseling. We refer to vulvar "care" rather than "hygiene" so as to avoid reinforcing the idea that there is a cleanliness issue. Proper care of vulvar skin is revisited at every related encounter (Box 2). We find both verbal counseling and written instructions helpful. Because the adoption of vulvar skin care recommendations and subsequent improvement of symptoms takes time, most of our patients (78\%) initially received a topical steroid for more rapid symptom control. Topical ointments are preferred over creams when possible since creams often contain skin irritants and allergens. Other common treatments include topical anti-fungals and estrogens, lidocaine gel, and hydroxyzine at bedtime, as clinically indicated.

Follow up encounters should revisit product use and re-emphasize proper vulvar care. For patients with refractory symptoms, additional testing (including biopsy) or consultation with a dermatologist should be considered. Referral to other medical specialties such as rheumatology or internal medicine may be helpful if a non-gynecologic primary disorder or condition is suspected.

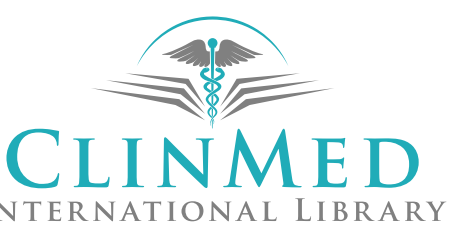

